\title{
Quelle définition et quelle stratification de la désescalade antibiotique ?
}

\author{
How Can Antibiotic De-Escalation Be Defined and Stratified?
}

\author{
J.-R. Zahar • E. Weiss $\cdot$ A. Tabah
}

Reçu le 5 décembre 2015 ; accepté le 22 décembre 2015

(C) SRLF et Lavoisier SAS 2015

La diffusion des mécanismes de résistance plasmidique au sein des entérobactéries (c'est-à-dire, diffusion des bêtalactamases à spectre élargi et des carbapénémases) et le rôle de l'antibiothérapie dans l'amplification de ce phénomène sont probablement à l'origine du regain d'intérêt quant à la désescalade antibiotique. En effet, l'augmentation de la prévalence de la résistance au sein des entérobactéries et notre incapacité en pratique clinique à identifier les patients infectés par des entérobactéries résistantes ont pour principale conséquence une surconsommation des antibiotiques dits de réserve (c'est-à-dire les carbapénèmes) [1].

Pour limiter les conséquences d'un tel phénomène, nous avons dans notre arsenal thérapeutique et diagnostique deux possibilités :

- mieux identifier les patients infectés par de tels microorganismes pour cibler nos choix antibiotiques ;

- et/ou réévaluer nos choix probabilistes une fois que les micro-organismes responsables et leur niveau de sensibilité sont connus. Depuis les années 1990, cette seconde option intitulée « désescalade », du fait de la gravité des malades de réanimation et de la nécessité d'éviter toute inadéquation [2], a été largement défendue et acceptée.

Alors même que la politique de désescalade est largement utilisée et évaluée dans des travaux nationaux et internationaux, il n'existe aucune définition consensuelle et pratique dans la littérature permettant de guider le clinicien au lit du malade [3]. D'un point de vue pratique, l'objectif de la

\footnotetext{
J.-R. Zahar ( $\bowtie)$

Infection Control Unit ; université d'Angers, CHU d'Angers, F-49000 Angers, France

e-mail : jrzahar@gmail.com

E. Weiss

AP-HP, hôpital Beaujon, Department of Anesthesiology and Critical Care, université Paris-Denis-Diderot, Clichy, France

\section{A. Tabah}

Intensive Care Unit, The Royal Brisbane and Women's Hospital, Burns, Trauma, and Critical Care Research Centre, the University of Queensland, Brisbane, Australia
}

désescalade serait de limiter l'émergence de la résistance à l'échelon individuel et collectif.

Plusieurs facteurs participent à l'émergence de la résistance. Outre l'acquisition de bactéries par transmission manuportée, il est important de souligner le rôle des items ci-après :

- le choix des molécules antibiotiques ;

- leur diffusion ;

- la durée de l'antibiothérapie ;

- leurs conséquences sur les microbiotes, spécifiquement sur le principal réservoir bactérien qui reste le microbiote digestif.

Il est important de souligner que les antibiotiques qui ont la meilleure diffusion digestive et le plus d'activité sur les bactéries anaérobies sont probablement ceux qui risquent le plus de favoriser l'émergence de résistance bactérienne [4].

En réanimation comme ailleurs, il n'existe pas de définition consensuelle de la désescalade antibiotique. Ainsi, les cliniciens et les chercheurs confondent fréquemment le spectre d'un antibiotique donné et ses conséquences sur les microbiotes. Par exemple, alors que la prescription des carbapénèmes expose à un risque d'émergence de bactéries naturellement résistantes ou ayant acquis des mécanismes de résistances [5], les conséquences liées à la prescription de cette molécule seront probablement équivalentes à celles liées à la prescription de l'association pipéracilline-tazobactam dans un monde de patients porteurs de bactéries résistantes. En effet, ces deux classes antibiotiques partagent les mêmes risques écologiques, dans la mesure où leur diffusion dans le tube digestif et leur activité sur les bactéries anaérobies sont élevées [4].

Cela est d'autant plus vrai pour les patients de réanimation, dans la mesure où la prescription antibiotique dans ce secteur vient se surajouter aux prescriptions antérieures préalables à l'admission [6] et que l'urgence d'un traitement approprié [2] induit les cliniciens à choisir des antibiotiques à très large spectre pour l'antibiothérapie probabiliste.

La désescalade a été définie selon les études comme étant la réduction du nombre d'antibiotiques comparativement à la 
prescription initiale probabiliste, l'arrêt des classes antibiotiques initialement prescrites et secondairement inutiles, la réduction du spectre antibiotique, la « rationalisation » des antibiotiques ou encore la réduction des durées des traitements. Certaines études ont même considéré que le relais par voie orale d'un antibiotique administré par voie intraveineuse constituait aussi une désescalade. De plus, il n'existe aucun consensus quant au délai de réalisation de cette réévaluation. Si généralement il est recommandé d'effectuer celle-ci à la $48^{\mathrm{e}}$ ou $72^{\mathrm{e}}$ heure, certains auteurs ont précisé que le délai de réalisation de la désescalade devait se situer entre le deuxième et le cinquième jour après l'initiation des antibiotiques.

Récemment, Leone et al. ont utilisé la notion d'antibiotique pivot (généralement une bêtalactamine) et la notion d'antibiotique " compagnon » (généralement un aminoglycoside ou une fluoroquinolone) pour définir la désescalade. Dans ce travail multicentrique effectué en France, la désescalade était définie comme la réduction du spectre de l'antibiotique pivot et l'arrêt au troisième jour de l'antibiotique compagnon ou des molécules initiées pour la couverture d'une éventuelle infection à bactéries multirésistantes et secondairement inutiles en l'absence de résistance bactérienne identifiée [7]. Plus récemment, deux travaux se sont intéressés à la définition de la désescalade en utilisant une méthode Delphi. Dans un premier travail, un groupe d'experts français s'intéressant aux bêtalactamines a proposé une définition en six classes permettant d'évaluer la désescalade. Cette définition était fondée sur le spectre et les conséquences écologiques des différentes molécules [8]. Madaras-Kelly et al. aux États-Unis [9] a développé un score prenant en compte le spectre antibactérien des différentes classes antibiotiques. Ces deux derniers travaux, au regard de leurs conclusions divergentes, soulignent la difficulté d'obtenir un consensus quant aux conséquences écologiques des antibiotiques et au poids des données écologiques locales, dans l'évaluation de ces conséquences. Ainsi, alors que dans l'étude de MadarasKelly et al. l'association pipéracilline-tazobactam (probablement du fait de la diffusion des entérocoques résistants à la vancomycine) était considérée comme celle ayant les conséquences écologiques les plus importantes, l'étude française considérait que c'était la classe des carbapénèmes en raison de leur spectre plus large et de leur rôle en tant que traitement de réserve.

Si la maîtrise de l'émergence de résistance reste l'objectif premier du contrôle de l'antibiothérapie, il est important de souligner qu'aucune étude en réanimation n'a à ce jour évalué le rôle de la désescalade sur le plan écologique. Les différentes études de la littérature se sont limitées à évaluer les conséquences de la désescalade sur la mortalité, la durée de séjour et rarement sur le nombre de journées passées sans antibiotique.
Pour redéfinir la désescalade, il nous paraît important de définir notre objectif premier. Celui-ci ne peut être ni la mortalité ni la durée de séjour des patients en réanimation, dans la mesure où de nombreux facteurs confondants interviennent dans la survenue de ces deux objectifs. Par ailleurs, il est impensable de croire que la politique de désescalade aura à court comme à moyen terme des conséquences sur l'incidence ou même la prévalence de la résistance. Ce dernier phénomène s'est installé au cours des 40 dernières années, et tout pousse à croire qu'il persistera malgré les efforts entrepris [10].

Il nous semble donc important de souligner que toute politique de désescalade devra avoir pour objectif de réduire la consommation globale des antibiotiques à l'échelon individuel et collectif. En effet, le volume de prescription antibiotique est un facteur majeur dans la survenue de la résistance, que ce soit à l'échelon individuel ou collectif. Chaque dose d'antibiotique est associée à un risque d'émergence de résistance, et il suffit d'une dose (quelle que soit la molécule choisie) pour que cette dernière s'exprime et soit visible [11].

En pratique, la désescalade antibiotique ne peut se limiter à la réévaluation d'une classe antibiotique, à l'arrêt d'une molécule ou encore à la durée de prescription d'un antibiotique donné. Elle est à la fois chacune de ces mesures et toutes réunies. À l'instar de l'hygiène hospitalière, qui ne peut se résumer par l'hygiène des mains ou par l'isolement des patients, la désescalade doit être à notre sens définie comme un fagot (bundle) de mesures introduites au sein d'une unité. Elle devra prendre en compte dans le choix des molécules probabilistes les données écologiques locales, en privilégiant les molécules dont les conséquences écologiques connues sont les plus limitées. Les durées antibiotiques devront se fonder sur les durées minimales identifiées dans la littérature et être ajustées en fonction de l'évolution clinique du patient, le tout permettant de définir le meilleur traitement individuel disponible. Notre objectif premier devra être une réduction des volumes de prescriptions antibiotiques à l'échelon de l'unité de réanimation et, à l'échelon individuel, la diminution du nombre de journées d'antibiothérapie en et hors réanimation. Cette politique devra à notre sens s'enrichir de la restriction de certaines molécules, dont les conséquences écologiques sont bien connues, mais qui devra certainement dépendre des données épidémiologiques propres à chacune de nos réanimations. Cette politique proposée est bien plus difficile à mettre en œuvre et à évaluer comparativement à l'exclusion d'une classe antibiotique donnée. Toutefois, nous souhaitons alerter les collègues sur le danger encore une fois de focaliser nos efforts sur l'accessoire au risque d'oublier l'essentiel et le fondamental.

Pour conclure, du point de vue du clinicien, la désescalade se résumerait aux trois actions suivantes : interrompre les traitements inutiles le plus rapidement possible (c'està-dire, dans les 48 premières heures suivant leur initiation), 
réduire les durées des traitements et tendre vers une durée de prescription individualisée. Enfin, choisir les molécules à spectre le plus étroit possible. Cette dernière action est probablement la plus difficile à définir en réanimation, alors même qu'elle ne nous semble pas à l'heure actuelle la plus urgente à introduire.

Liens d'intérêts : Jean-Ralph Zahar a reçu des honoraires des laboratoires MSD. E. Weiss et A. Tabah déclarent n'avoir aucun lien d'intérêt.

\section{Références}

1. Prinapori R, Guinaud J, Khalil A, et al (2013) Risk associated with a systematic search of extended-spectrum $\beta$-lactamase-producing Enterobacteriaceae. Am J Infect Control 41:259-60

2. Zahar JR, Timsit JF, Garrouste-Orgeas M, et al (2011) Outcomes in severe sepsis and patients with septic shock: pathogen species and infection sites are not associated with mortality. Crit Care Med 39:1886-95

3. Tabah A, Cotta M, Garnacho-Montero J, et al (2016) A systematic review of the definitions, determinants and clinical outcome of antimicrobial de-escalation in the intensive care unit. Clin Infect Dis XXX
4. Sullivan A, Edlund C, Nord CE (2001) Effect of antimicrobial agents on the ecological balance of human microflora. Lancet Infect Dis 1:101-14

5. Lepper PM, Grusa E, Reichl H, et al (2002) Consumption of imipenem correlates with beta-lactam resistance in Pseudomonas aeruginosa. Antimicrob Agents Chemother 46:2920-5

6. Thomas Z, Bandali F, Sankaranarayanan J, et al (2015) A multicenter evaluation of prolonged empiric antibiotic therapy in adult ICUs in the United States. Crit Care Med 43:2527-34

7. Leone M, Bechis C, Baumstarck K, et al (2014) De-escalation versus continuation of empirical antimicrobial treatment in severe sepsis: a multicenter non-blinded randomized noninferiority trial. Intensive Care Med 40:1399-408

8. Weiss E, Zahar JR, Lesprit P, et al (2015) "De-escalation" Study Group; "De-escalation" Study Group. Elaboration of a consensual definition of de-escalation allowing a ranking of $\beta$-lactams. Clin Microbiol Infect 21:649.e1-649.e10

9. Madaras-Kelly K, Jones M, Remington R, et al (2014) Development of an antibiotic spectrum score based on veterans affairs culture and susceptibility data for the purpose of measuring antibiotic de-escalation: a modified Delphi approach. Infect Control Hosp Epidemiol 35:1103-13

10. Holmes AH, Moore LS, Sundsfjord A, et al (2016) Understanding the mechanisms and drivers of antimicrobial resistance. Lancet 387:176-87

11. Armand-Lefèvre L, Angebault C, Barbier F, et al (2013) Emergence of imipenem-resistant Gram-negative bacilli in intestinal flora of intensive care patients. Antimicrob Agents Chemother $57: 1488-95$ 